\title{
Síndroma de Takotsubo em medicina geral e familiar: a propósito de um caso clínico
}

Sofia Faria*, Cátia Cordeiro**, Luís Filipe Cavadas***

\section{RESUMO}

Introdução: A síndroma de Takotsubo caracteriza-se por uma disfunção sistólica transitória do ventrículo esquerdo, que mimetiza o enfarte agudo do miocárdio, na ausência de doença coronária significativa. É mais comum em mulheres na pós-menopausa, com suspeita de síndroma coronário agudo (SCA) e manifestações clínicas e eletrocardiográficas desproporcionais relativamente aos marcadores cardíacos. Um trigger emocional ou físico está muitas vezes presente, podendo nestes casos o médico de família (MF) desempenhar um papel importante no seu diagnóstico e seguimento.

Descrição do caso: Apresenta-se o caso de uma doente de 49 anos, admitida no serviço de urgência por suspeita de SCA, apresentando dor torácica, dispneia e náuseas. Foi constatada insuficiência cardíaca (IC) aguda, com compromisso severo da função sistólica do ventrículo esquerdo, no entanto, na ausência de doença coronária. A evolução clínica foi favorável, com recuperação completa da função cardíaca, tendo sido feito o diagnóstico de síndroma de Takotsubo. Neste contexto, com o conhecimento adquirido que tinha desta doente, o MF conseguiu identificar o stress laboral e familiar como provável fator desencadeante da síndroma, intervindo de forma importante no ensino da gestão da ansiedade e no seu seguimento.

Comentário: Após o episódio de urgência descrito, a doente manteve-se assintomática do foro cardiovascular, sendo o tratamento essencialmente de suporte. Assim, caberá ao MF o controlo sintomático da ansiedade e do foro emocional, assim como a vigilância de sinais e/ou sintomas de IC. O MF desempenhará um papel de prevenção, no sentido de identificar e atuar precocemente em possíveis fatores desencadeantes, promovendo e reforçando a importância da adoção de hábitos e estilos de vida saudáveis e ainda na monitorização do estado de saúde da utente, no seu contexto biopsicossocial, de forma a evitar e minimizar complicações a longo prazo.

Palavras-chave: Síndroma de Takotsubo; Cardiomiopatia; Cuidados de saúde primários; Cardiologia.

\section{INTRODUÇÃO}

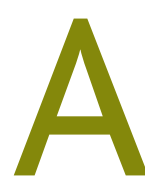
síndroma Takotsubo, também denominada cardiomiopatia induzida pelo stress ou síndroma do coração partido, caracteriza-se por uma disfunção sistólica transitória do ventrículo esquerdo (VE), que mimetiza o enfarte agudo do miocárdio (EAM) na ausência de doença coronária significativa. ${ }^{1-3}$ Estima-se que a sua prevalência atinja 1 a $2 \%$ dos casos suspeitos de síndroma coronária aguda (SCA), ${ }^{1,3-4}$ sendo mais comum nas mulheres, sobretudo

\footnotetext{
*Interna de Medicina Geral e Familiar de Medicina Geral e Familiar. USF Lagoa, Unidade Local de Saúde de Matosinhos.

**Interna de Formação Específica de Medicina Geral e Familiar. USF Lagoa, Unidade Local de Saúde de Matosinhos.

***Assistente de Medicina Geral e Familiar. USF Lagoa, Unidade Local de Saúde de Matosinhos
}

no período pós-menopausa. ${ }^{1}$ Deverá suspeitar-se deste diagnóstico perante manifestações clínicas e eletrocardiográficas sugestivas de EAM, desproporcionais relativamente aos marcadores cardíacos. Um trigger emocional ou físico está muitas vezes presente. ${ }^{1}$

A descrição deste caso clínico pretende colocar em evidência um diagnóstico raro para um quadro sintomático comum, cuja manifestação inicial poderá muitas vezes surgir no âmbito dos cuidados de saúde primários, e enfatizar a atuação do médico de família (MF) no seu diagnóstico e orientação.

\section{DESCRIÇÃO DO CASO}

Apresenta-se o caso de uma doente do sexo feminino, de raça caucasiana, com 49 anos de idade, natural de Angola e divorciada. 
Inserida numa família alargada, classe III segundo a classificação de Graffar, com relações familiares e laborais conflituosas, geradoras de stress e ansiedade.

Como antecedentes familiares relevantes apresenta hipertensão arterial, um fator de risco partilhado por ambos os progenitores, bem como diabetes mellitus, presente na mãe.

Como antecedentes pessoais mais significativos salienta-se a dislipidemia (colesterol LDL 144,8mg/dL), o excesso de peso (índice de massa corporal $27 \mathrm{~kg} / \mathrm{m}^{2}$ ), insuficiência venosa periférica, esofagite de refluxo, gastrite crónica e hérnia do hiato esofágico e uma perturbação de ansiedade. Trata-se de uma utente com hábitos tabágicos no passado (cerca de 10 unidades maço-ano) e também hábitos passados de consumo etílico abusivo (cerca de 80g/dia), encontrando-se atualmente abstinente. À data do caso relatado, a utente encontrava-se medicada apenas com um inibidor da bomba de protões e realizava contraceção hormonal oral combinada.

Em setembro de 2014, a utente recorre ao serviço de urgência hospitalar com um quadro de dor retroesternal, do tipo moedeira, de intensidade moderada, com irradiação cervical, acompanhada de dispneia e náuseas. A dor mantinha-se constante, sem fatores de alívio ou agravamento.

À admissão encontrava-se pálida, taquipneica e taquicárdica, apresentando à auscultação cardiopulmonar sons respiratórios diminuídos basalmente e crepitações nos terços inferiores de ambos os campos pulmonares. É realizado de imediato um eletrocardiograma (ECG) que revela inversão da onda T nas derivações precordiais de V2 a V6, estando em ritmo sinusal. A radiografia torácica mostra congestão pulmonar, atingindo a metade inferior de ambos os campos pulmonares. Analiticamente há uma elevação dos marcadores cardíacos, principalmente da troponina I $(1,646 \mathrm{ng} / \mathrm{mL}$; valor de referência $(\mathrm{VR})<0,0329 \mathrm{ng} / \mathrm{mL})$, mas também da CK-MB (11,3ng/mL; VR $<6,4 \mathrm{ng} / \mathrm{mL})$, de D-dímeros (773ng/mL; Positivo > 500ng/mL) e do BNP $(1222 \mathrm{pg} / \mathrm{mL} ; \mathrm{VR}<100 \mathrm{pg} / \mathrm{mL})$. Assumido o diagnóstico de uma síndroma coronária aguda (SCA) sem elevação do segmento ST, a utente é transferida para a Unidade de Cuidados Intensivos Polivalente, iniciando terapêutica com antiagregante plaquetário (ácido acetilsalicílico), diurético e nitratos. É realizado um eco- cardiograma, que revela uma insuficiência cardíaca (IC) aguda, com sinais de insuficiência tricúspide moderada a grave e compromisso grave da função sistólica do VE, com fração de ejeção muito diminuída (FEVE 25\%). Constata-se ainda acinésia dos segmentos médio e apical do septo, paredes anterior e lateral, e ainda hipocinésia do segmento apical da parede inferior do VE com dilatação aneurismática a esse nível.

É realizada coronariografia que não revelou doença coronária significativa, sendo assumido o diagnóstico de IC aguda em doente jovem, de causa não esclarecida, com compromisso grave da função do VE. Durante o restante internamento, a doente manteve a estabilidade hemodinâmica, apresentando uma evolução favorável e rápida melhoria do quadro, com recuperação completa da função cardíaca.

A restante investigação laboratorial, incluindo serologias de sífilis, vírus da imunodeficiência humana (VIH), vírus da hepatite B (VHB), vírus da hepatite C (VHC) foi negativa; também as serologias de vírus de Epstein Barr (VEB), vírus herpes simples 1 e 2 (VHS 1 e 2) e parvovírus foram negativas; a função tiroideia demonstrou-se dentro dos valores da normalidade; os doseamentos de ferro, ferritina, vitamina B12 e folatos foram normais; a pesquisa de anticorpos ANA e anti-dsDNA e de fator reumatoide foi negativa; a análise do complemento e eletroforese de proteínas séricas não apresentou alterações de relevo. Verificou-se que não existia história prévia de síndroma gripal ou aparente stress emocional recente, assim como manifestações de doença autoimune, nem história familiar de miocardiopatias.

A doente teve alta ao final de nove dias de internamento, mantendo-se assintomática, sem ocorrência de novos episódios de dor torácica e sem sinais de IC agudizada. Não foi esclarecida a etiologia da IC aguda, apesar do estudo realizado durante o internamento, sendo a doente orientada para consulta externa de cardiologia. À data da alta, a seguinte terapêutica foi instituída: lisinopril 5mg (uma vez por dia), carvedilol $3,125 \mathrm{mg}$ (duas vezes por dia), espironolactona $12,5 \mathrm{mg}$ (uma vez por dia), pantoprazol $20 \mathrm{mg}$ (uma vez por dia) e rosuvastatina 10mg (uma vez por dia).

Após o episódio de internamento, a utente recorreu ao seu MF, dando conhecimento do ocorrido. Na consulta apresentava-se assintomática do foro cardiovas- 
cular e sem alterações objetiváveis ao exame físico; no entanto, encontrava-se muito angustiada, com receios e medos relativos ao episódio ocorrido, tendo sido alvo de escuta terapêutica, assim como de aconselhamento e educação para a saúde ao longo das várias consultas de seguimento nos cuidados de saúde primários, enquanto aguardava pela consulta de cardiologia.

Com o conhecimento adquirido que o MF tinha da utente percebeu que provavelmente o fator desencadeante desta patologia, neste contexto em particular, teria sido um stress emocional motivado pelo trabalho. A utente trabalhava num cabeleireiro e referia conflitos diários com as colegas de trabalho, estando numa semana particularmente difícil, com muito trabalho e, consequentemente, com maior stress profissional. Neste processo, o MF trabalhou a ansiedade e o stress emocional, agendando consultas mais frequentes, inicialmente com periodicidade semanal e posteriormente mensal, onde a escuta terapêutica teve um papel muito importante no ensino da gestão da ansiedade.

Durante o seguimento por cardiologia, a utente realizou novo ecocardiograma que demonstrou a completa recuperação da função cardíaca, apresentando a função sistólica de ambos os ventrículos conservada, com uma fração de ejeção do VE estimada em $66 \%$, sem alterações segmentares evidentes e sem alterações morfológicas significativas das estruturas valvulares. Foi também realizada uma ressonância magnética cardíaca que excluiu qualquer alteração estrutural cardíaca.

A utente teve alta de cardiologia em fevereiro de 2016, com o diagnóstico final de síndroma de Takotsubo. Foi mantida a terapêutica instituída inicialmente à data do internamento, tendo sido dada indicação para manutenção da vigilância no seu MF, embora sem orientação escrita ou verbal de cuidados específicos.

O MF continuou a intervir, de forma a esclarecer, tranquilizar e vigiar esta utente nesta fase complicada e causadora de ansiedade, instabilidade emocional e incapacidade laboral. Realizou também uma avaliação familiar. Salienta-se um contexto de disfunção familiar grave, com conflitos frequentes entre o agregado familiar, importantes geradores de stress que, tendo por base a personalidade ansiosa da utente, poderão ter contribuído para o despoletar desta síndroma. No momento da avaliação, a utente demonstrou apresentar-se já com humor estabilizado, tendo sido, no entanto, um mo- mento importante de enfatização de mecanismos de coping apropriados para a gestão de conflitos, importante para prevenir a recorrência do quadro clínico.

\section{COMENTÁRIO}

Vários mecanismos fisiopatológicos têm sido propostos para explicar a síndroma de Takotsubo, entre os quais se incluem a doença aterosclerótica oculta, a ocorrência de um espasmo de multivasos ou a disfunção microvascular. ${ }^{1}$ No entanto, a hipótese mais aceite atualmente é a do excesso de catecolaminas que, por sobrecarga de cálcio nos miócitos cardíacos, leva a uma perturbação transitória da contração e função ventriculares. ${ }^{1-2}$

O stress emocional pode estar envolvido, estando o MF numa posição incomparável para compreender o contexto biopsicossocial e familiar dos seus utentes e, assim, possivelmente identificar eventuais fatores de risco, que poderão estar na base desta síndroma.

Relativamente aos exames auxiliares de diagnóstico (EAD), as alterações eletrocardiográficas sugestivas de EAM são comuns, sendo a mais frequente a elevação do segmento ST. ${ }^{1,4}$ Os marcadores cardíacos apresentam geralmente uma ligeira elevação, verificando-se que a troponina sérica está elevada na maioria destes doentes e os níveis de CK estão geralmente normais ou ligeiramente aumentados. Também os níveis do peptídeo natriurético (BNP) estão elevados em grande parte destes doentes. ${ }^{1} \mathrm{O}$ ecocardiograma demonstra geralmente um compromisso da função sistólica do VE, sendo a coronariografia imprescindível, no sentido de excluir a presença de doença coronária significativa. ${ }^{1,3}$ A ressonância magnética cardíaca pode ser útil para o diagnóstico e avaliação da miocardiopatia, sobretudo para exclusão de doença cardíaca estrutural. ${ }^{1,4}$

Apresentou-se um caso clínico de uma síndroma de Takotsubo numa doente de 49 anos de idade, com dois fatores de risco cardiovasculares identificados: a dislipidemia e o excesso de peso. Era também do conhecimento do seu MF o stress emocional marcado, envolvendo o contexto sociofamiliar da utente. Tratou-se de um caso típico desta síndroma, com recuperação total da função cardíaca, de forma rápida e sem deixar sequelas, tendo-se a doente mantido assintomática após o evento e sem novos episódios de dor torácica ou sinais de IC. Após um período de tempo em seguimento 
por cardiologia, confirmado o diagnóstico e não havendo doença cardíaca estrutural, a doente teve alta com orientação para manutenção da vigilância pelo seu MF.

Pretende-se com este caso clínico enfatizar a importância do MF na gestão desta patologia, bem como a importância de uma boa articulação entre os cuidados de saúde primários e secundários. De facto, o contacto com o MF pode ser de grande auxílio na abordagem diagnóstica e no diagnóstico diferencial desta síndroma, tendo em conta o conhecimento abrangente que este detém do utente, do seu contexto biopsicossocial e da relação singular que estabelece com os seus utentes através dos cuidados de saúde longitudinais prestados.

Por outro lado, após o diagnóstico definitivo da síndroma de Takotsubo, estando a utente assintomática e apresentando uma função cardíaca normal, surgiu a dúvida na sua orientação relativamente à medicação de suporte previamente instituída (lisinopril, carvedilol, espironolactona). Estas questões colocaram-se ao MF da utente aquando da alta de cardiologia. A existência de informação clínica de retorno é fundamental para que exista de facto uma articulação de cuidados eficaz, que se traduzirá certamente numa melhoria da qualidade de cuidados de saúde e numa maior satisfação por parte dos utentes e profissionais de saúde.

De facto, o tratamento destes doentes é essencialmente de suporte, desempenhando aqui um papel muito importante as consultas do MF. Tratando-se de uma síndroma induzida por catecolaminas, devem instituir-se um $\beta$-bloqueador e um inibidor da enzima de conversão da angiotensina (IECA) até à recuperação da função cardíaca, sendo que nos casos em que esta é completa não se justifica a manutenção do IECA. Por sua vez, considerando o mecanismo fisiopatológico mais aceite para esta síndroma, poderá manter-se o $\beta$-bloqueador. Os diuréticos são recomendados em casos de IC e a anticoagulação a curto prazo é indicada em doentes com fibrilhação auricular ou formação de trombos. ${ }^{1}$

A maioria dos doentes apresenta um excelente prognóstico, com uma história natural aparentemente benigna e recuperação integral da função ventricular. ${ }^{5} \mathrm{Os}$ sintomas, as alterações eletrocardiográficas, os marcadores cardíacos e anomalias de movimento ventricula- res desaparecem geralmente entre seis a oito semanas. ${ }^{6-7}$ A mortalidade intra-hospitalar é baixa (inferior a 2\%), sendo as complicações mais comuns a IC sistólica e a rutura cardíaca. Nos doentes com recuperação completa verifica-se uma sobrevida a longo prazo semelhante à da população geral, sendo a taxa de recorrência desta síndroma inferior a $10 \%{ }^{1,8}$

Assim, e a longo prazo, caberá ao MF o controlo sintomático da ansiedade e do foro emocional, a vigilância de sinais e/ ou sintomas de IC, assim como a sua devida orientação terapêutica, sendo esta a principal complicação possível conhecida desta síndroma.

Ainda do ponto de vista do MF, será muito pertinente, tendo em conta o acompanhamento do utente ao longo do seu ciclo vital, assim como o conhecimento prévio do seu contexto familiar e biopsicossocial, uma abordagem holística que inclua a monitorização do estado de saúde global, incluindo o estado de saúde mental, para uma intervenção atempada e apropriada, caso se demonstre necessário. Além disso, aproveitando o privilégio de ser também o médico «da família»e, assim, conhecer e acompanhar os outros elementos familiares, abre-se uma porta para uma abordagem não apenas individual, mas de toda a dinâmica familiar, que deverá ser alvo de monitorização e intervenção adequada, uma vez que esta se identificou como um fator que condicionou uma desregulação emocional da paciente, conduzindo ou contribuindo para esta síndroma.

Trabalhar a família é importante, pois permite dar resposta às dúvidas dos restantes elementos familiares que vivem de perto esta situação, receosos de novos episódios e de como proceder em família.

De facto, na síndroma de Takotsubo está muitas vezes descrito um fator físico ou emocional significativo como fator despoletante, o que na maioria dos casos será um evento agudo e dificilmente previsível. Particularmente no caso clínico descrito, considerando os antecedentes de patologia ansiosa, será fundamental a sua vigilância regular a longo prazo no contexto dos CSP, antevendo-se uma possível abordagem farmacológica e/ou não farmacológica em caso de recidiva da perturbação ansiosa. Assim, na abordagem da síndroma de Takotsubo, o MF desempenhará um papel de prevenção, no sentido de identificar e atuar precocemente em possíveis fatores desencadeantes, promovendo e reforçando a importância da adoção de hábi- 
tos e estilos de vida saudáveis e ainda na monitorização do estado de saúde do utente no seu contexto biopsicossocial, de forma a evitar e minimizar complicações a longo prazo.

\section{REFERÊNCIAS BIBLIOGRÁFICAS}

1. Nóbrega S, Brito D. Miocardiopatia Takotsubo: estado da arte [The 'broken heart syndrome': state of the art]. Rev Port Cardiol. 2012;31(9):589-96.

2. Richard C. Stress-related cardiomyopathies. Ann Intensive Care. 2011; 1:39.

3. Gianni M, Dentali F, Grandi AM, Sumner G, Hiralal R, Lonn E. Apical ballooning syndrome or takotsubo cardiomyopathy: a systematic review. Eur Heart J. 2006;27(13):1523-9.

4. Koulouris $S$, Pastromas $S$, Sakellariou D, Katrimenos T, Piperopoulos $P$, Manolis AS. Takotsubo cardiomyopathy: the 'broken heart' syndrome. Hellenic J Cardiol. 2010;51(5):451-7.

5. Ionescu CN, Aguilar-Lopez CA, Sakr AE, Ghantous AE, Donohue TJ. Longterm outcome of Tako-tsubo cardiomyopathy. Heart Lung Circ. 2010;19(10):601-5.

6. Madhavan M, Prasad A. Proposed Mayo Clinic criteria for the diagnosis of Tako-Tsubo cardiomyopathy and long-term prognosis. Herz. 2010 35(4):240-3.
7. Stöllberger C, Finsterer J, Schneider B. Transient left ventricular dysfunction (tako-tsubo phenomenon): findings and potential pathophysiological mechanisms. Can J Cardiol. 2006;22(12):1063-8.

8. Opolski G, Pawlak MM, Roik MF, Kochanowski J, Scislo P, Piatkowski R, et al. Clinical presentation, treatment, and long-term outcomes in patients with takotsubo cardiomyopathy: experience of a single cardiology center. Pol Arch Med Wewn. 2010;120(6):231-6.

\section{CONFLITO DE INTERESSES}

Os autores declaram não ter quaisquer conflitos de interesse.

\section{FINANCIAMENTO DO ESTUDO}

Os autores declaram que o trabalho relatado neste manuscrito não foi objecto de qualquer tipo de financiamento externo.

\section{ENDEREÇO PARA CORRESPONDÊNCIA \\ Sofia Faria \\ E-mail: faria.sofia@hotmail.com \\ https://orcid.org/0000-0003-1034-7094}

Recebido em 17-03-2017

Aceite para publicação em 13-06-2018

\section{ABSTRACT}

\section{TAKOTSUBO SYNDROME IN FAMILY MEDICINE: A CASE REPORT}

Introduction: Takotsubo syndrome is a transient systolic dysfunction of the left ventricle, which mimics acute myocardial infarction in the absence of coronary artery disease. It is more common in postmenopausal women with suspected acute coronary syndrome (ACS) and electrocardiographic abnormalities that are not proportional to the values of cardiac markers. An emotional or physical trigger is often present, and in these cases the family physician can play an important role in the diagnosis and follow-up.

Case study: The authors describe the case of a 49-year-old woman admitted to the emergency room with suspected ACS, presenting with chest pain, dyspnea and nausea. Acute heart failure was observed, with a severe compromise of left ventricular systolic function, but in the absence of coronary disease. A favorable clinical evolution was observed, with a complete recovery of cardiac function, and the diagnosis of Takotsubo syndrome was made. In this context, the general practitioner (GP) knowledge about this patient was able to identify work and family stress as probable triggers of this syndrome, and played an important role on anxiety management and follow-up.

Comment: After this event, the patient remained asymptomatic, and was essentially given general supportive care. Thus, the GP will be responsible for the symptomatic control of anxiety and emotional disorders, as well as for the monitoring of signs and/or symptoms of heart failure. The GP will play a preventive role, by identifying and acting timely on putative triggers, promoting and reinforcing the importance of adopting healthy habits and lifestyles, and monitoring the patient's biopsychosocial health status, in order to avoid and minimize long-term complications.

Keywords: Takotsubo syndrome; Cardiomyopathy; Primary health care; Cardiology. 